\title{
Techical paper
}

\section{Measuring Cheese Maturation with the Fluorescence Fingerprint}

\author{
Mito KokawA ${ }^{1,2}$, Shoma Ikegami ${ }^{3}$, Akira ChibA ${ }^{3}$, Hiroshi Koishinara ${ }^{3}$, Vipavee Trivittayasil ${ }^{4}$, Mizuki Tsuta ${ }^{4 *}$, \\ Kaori Fusita ${ }^{4}$ and Junichi SugIYAma ${ }^{4}$
}

\author{
${ }^{I}$ Research Fellow of Japan Society for the Promotion of Science, 5-3-1 Koujimachi, Chiyoda-ward, Tokyo 102-0083, Japan \\ ${ }^{2}$ Analytical Science Division, National Food Research Institute, National Agriculture and Food Research Organization, 2-1- \\ 12 Kan-nondai, Tsukuba, Ibaraki 305-8642, Japan \\ ${ }^{3}$ Food Research \& Development Institute, Morinaga Milk Industry Co., Ltd., 1-83-5, Higashihara, Zama, Kanagawa 252- \\ 8583, Japan \\ ${ }^{4}$ Food Engineering Division, National Food Research Institute, National Agriculture and Food Research Organization, 2-1- \\ 12 Kan-nondai, Tsukuba, Ibaraki 305-8642, Japan
}

Received February 7, 2015 ; Accepted April 28, 2015

The objective of this research was to develop a method using the fluorescence fingerprint (FF) to measure cheese maturation. FFs of sixteen cheese samples with maturation periods of 7 to 329 days were measured with 8 replicates for each sample, along with reference values of proteolysis index (ratio of soluble to total nitrogen) and total free amino acids. A partial least-squares (PLS) model to predict the maturation time, proteolysis index and free amino acids from the FF data was constructed. The coefficient of determination for cross-validation $\left(R^{2} \mathrm{CV}\right)$ was $0.93,0.79$, and 0.90 for the three indices, respectively. From the loadings of the PLS model, it was found that emission spectra at $345 \mathrm{~nm}$ excitation, attributable to oxidized lipids and Maillard reaction products, increased in intensity with prolonged maturation time.

Keywords: proteolysis index, free amino acids, PLS regression, Maillard products, oxidized lipid

\section{Introduction}

Processed cheese is produced by heating together natural cheese of different degrees of maturity with emulsifying salts and other dairy and non-dairy ingredients. It is consumed widely in Japan due to its mild flavor and versatility, and as of the year 2012, $43 \%$ of the cheese consumed in Japan was processed cheese (Ministry of Agriculture, 2013).

Properties of processed cheese are influenced by various chemical and compositional properties of the natural cheese such as fat, moisture and intact casein content (Kapoor and Metzger, 2008). Whereas in a young, unripe cheese, casein molecules are intact and can create a rigid structure in the processed cheese, the casein molecules in ripened cheese become hydrolyzed, yielding a softer product. A typical industrial processed cheese is produced from $30 \%$ young cheese, 50\% semi-mature cheese and 20\% mature cheese (Piska and Štětina, 2004), but since the crude natural cheese always varies in its degree of maturation, the mixing ratio requires adjustment to obtain a product of consistent quality.

Although multiple methods have been developed to measure cheese maturation such as gel electrophoresis and immunoblotting (Addeo et al., 1995), determination of water soluble nitrogen ratio (Lee et al., 1990) and by small scale processing using a rapid visco analyzer (RVA) (Kapoor et al., 2004), none of these are sufficiently quick and easy to use at the manufacturing level. Consequently, many processed cheese makers rely on their experience to adjust the ingredient blend for a specific product (Kapoor and Metzger, 
2008). The objective of this study is to develop a quick and objective method to measure the degree of cheese maturation, which would greatly aid in optimizing the manufacturing process.

Fluorescence has been used intensively to measure cheese properties such as texture (Dufour et al., 2001), oxidation (Andersen et al., 2005; Karoui et al., 2007), geographic origin (Karoui et al., 2004) and maturation (Herbert et al., 2000; Mazerolles et al., 2001). The range of research has expanded greatly with the development of front-face fluorescence, where the sample is placed at an acute angle with the incident light and reflected fluorescence is measured, enabling the measurement of turbid and solid samples (Dufour and Riaublanc, 1997). Cheese contains multiple constituents that are fluorescent, such as aromatic amino acids (Mazerolles et al., 2001), vitamin A (Herbert et al., 2000) and riboflavin (Andersen et al., 2005).

Recently, multiple studies have used the fluorescence fingerprint $(\mathrm{FF})$, also referred to as the excitation-emission matrix (EEM), to acquire more comprehensive information about a cheese sample (Andersen et al., 2005; Christensen et al., 2003). FF is a set of fluorescence spectra acquired at consecutive excitation wavelengths, creating a three-dimensional diagram (Jiang et al., 2010). The pattern of this diagram is unique for every constituent, rather like a fingerprint. The FF has an advantage over conventional fluorescence spectra because it includes emission spectra excited at many different excitation wavelengths, making it possible to measure complex samples that contain many fluorophores. Several studies using the FF on various target foods have been reported (Sadecka and Tothova, 2007) such as meat (Moller et al., 2003), olive oil (Guimet et al., 2004), wine (Yin et al., 2009) and beer (Sikorska et al., 2008).

Since the maturation process of cheese is very complex, with concurrent changes occurring to proteins, lipids and other constituents, a non-targeted approach such as the FF would be well suited for its measurement. In addition, FF measurement only takes a few minutes, requires no preprocessing steps for the sample such as extraction and degradation, and can potentially be performed non-destructively. Although multiple studies have used fluorescence to monitor the change in cheese during maturation (Dufour et al., 2000; Herbert et al., 2000; Karoui et al., 2007; Mazerolles et al., 2001), to our knowledge no study has measured the degree of maturation quantitatively. Therefore, in this study we aim to develop a quantitative measurement method using the FF.

\section{Materials and Methods}

Cheese samples Sixteen cheddar cheese samples were obtained for measurement. The cheese samples were produced in Hokkaido, Japan between October 2012 and November 2013, and matured for a period ranging from 7 to 329 days. Each cheese sample was divided into a block used for FF measurement and a block for reference chemical measurement. The blocks of cheese used for chemical measurements were frozen within two days of the FF measurement to stop further maturation.

FF measurement The blocks of cheese for FF measurement were cut into slabs approximately $1 \mathrm{~cm}$ in thickness, cored with a sharp stainless tube to produce a cylindrical shape with a diameter of $0.9 \mathrm{~cm}$, and sliced carefully to approximately $1.5 \mathrm{~mm}$ thickness with a microtome blade. This disk-shaped sample fitted the sample holder designed to measure samples in solid or powder form at a front-face configuration in the spectrophotometer (F7000; Hitachi High-Technologies corporation, Tokyo, Japan). The sample holder had a quartz-glass window, and the cheese samples were pushed against the quartz-glass so there was no air between the sample and the quartz glass.

The FFs of the samples were obtained with the spectrophotometer in the wavelength range of $200-700 \mathrm{~nm}$ for both excitation and emission wavelengths, with an interval of $5 \mathrm{~nm}$. The slit width of the monochrometer was $5 \mathrm{~nm}$ for both excitation and emission light, photomultiplier voltage was set at $460 \mathrm{~V}$, scanning speed was $60,000 \mathrm{~nm} / \mathrm{min}$ and response time was $0.002 \mathrm{sec}$. The spectrophotometer was calibrated in advance with rhodamine solution. Eight replicates were measured for each cheese sample.

Reference chemical measurement The proteolysis index (ratio of water-soluble nitrogen to total nitrogen) and total amount of free amino acids were measured as reference values for the degree of cheese maturation. The proteolysis index is defined as the ratio of soluble nitrogen in a $\mathrm{pH} 4.6$ buffer solution against the total amount of nitrogen (Kuchroo and Fox, 1982). Free amino acids were measured by the post-column ninhydrin method, using an amino acid detector (L-8900; Hitachi High Technologies) with \#2623PF column.

PLS model for prediction of maturation indices Three partial least-squares (PLS) regression models were constructed to predict maturation time, proteolysis index and total free amino acids from the FF data. Multivariate analysis was conducted using PLS Toolbox version 7.9.2 (Eigenvector Inc., USA) in MATLAB 7.12.0 (R2011a) software (MathWorks Inc., USA).

The FFs were preprocessed following the procedure of Yoshimura et al. (2014). First, data whose excitation wavelength was longer than that of the emission wavelength was removed, since fluorescence is defined as light emission with longer wavelengths than the excitation light. Next, the first-, second- and third-order scattered lights, which occurred at wavelengths where the emission wavelength was equal to, twice, and three times the excitation wavelength, respectively (Fujita et al., 2010), were removed. Finally, the wavelength conditions where excitation wavelengths were shorter than $230 \mathrm{~nm}$ or longer than $500 \mathrm{~nm}$ were removed, as there was significant noise that would increase the estimation error. The remaining data consisted of 3017 combinations of excitation and emission wavelengths.

Since the number of samples (16 samples $\times 8$ replicates) was too small to separate into calibration and validation groups, crossvalidation was used. Cross-validation executes several sub- 
validation calculations, each of which involves a different calibration and validation dataset, and the average prediction accuracy of all the sub-validation sets are calculated. The crossvalidation method was designed so that samples with the smallest and largest reference values were always included in the calibration set to avoid extrapolation (Trivittayasil et al., 2015), and replicate measurements of the same sample were all either in the calibration or validation set to avoid over-fitting.

Three pre-processing methods were used: mean-centering, normalization followed by mean-centering, and auto-scale. Normalization scaled the data so that the area underneath each spectrum equaled one, and auto-scale scaled each wavelength to zero mean and unit variance. The number of latent variables for each PLS model was determined by using the knee-finding algorithm in PLS Toolbox where a drop known as the "knee" is located in the scree plot (Henry et al., 1999).

\section{Results}

Changes occurring during maturation Figure 1 shows the change in proteolysis index and total free amino acids during cheese maturation. Generally, as the cheese matures, proteins break down into smaller peptides and amino acids, increasing the proteolysis index and the total amount of free amino acids. However, these values are not only dependent on the maturation time but are known to also be affected by fat content (Fenelon et al., 2000), temperature, salting, $\mathrm{pH}$ of the curd and humidity (Jin and Park, 1995). Consequently, the proteolysis index and total free amino acids could not be sufficiently predicted solely from maturation time. Therefore, three different prediction models were constructed for each of the three maturation indices: maturation time, proteolysis index and total free amino acids.

PLS prediction model Table 1 shows the prediction accuracy of the PLS models with different pre-processing methods. The model with the highest coefficient of determination for crossvalidation $\left(\mathrm{R}^{2} \mathrm{CV}\right)$ and lowest root mean square error of cross validation (RMSECV) was considered the most accurate. Preprocessing with normalization and mean-center gave the most accurate results for the prediction of maturation time and total free amino acids, whereas auto-scale gave the most accurate results for proteolysis index. Root mean square error of calibration (RMSEC) is a measure of the accuracy of a PLS model where all the samples are used in the calibration set. $\mathrm{R}^{2} \mathrm{CV}$, RMSECV and RMSEC were calculated from the average predicted values of the eight replicate measurements.
(A)

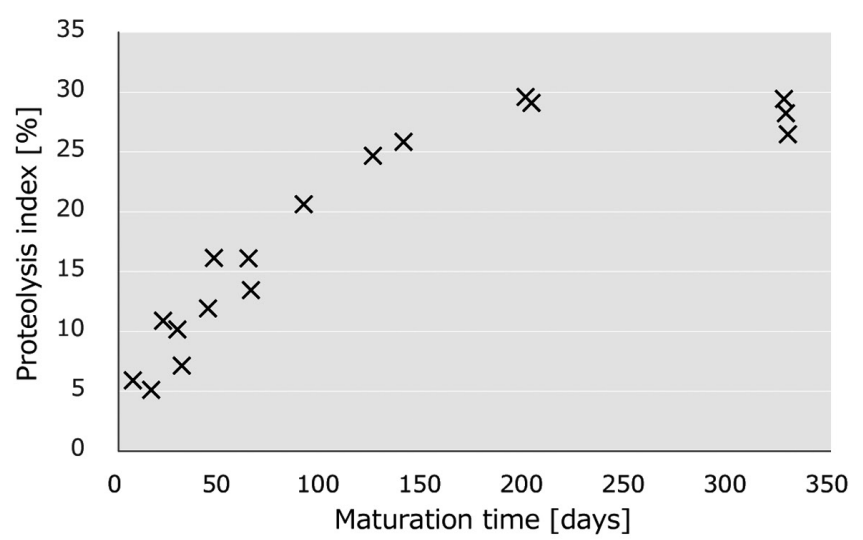

(B)

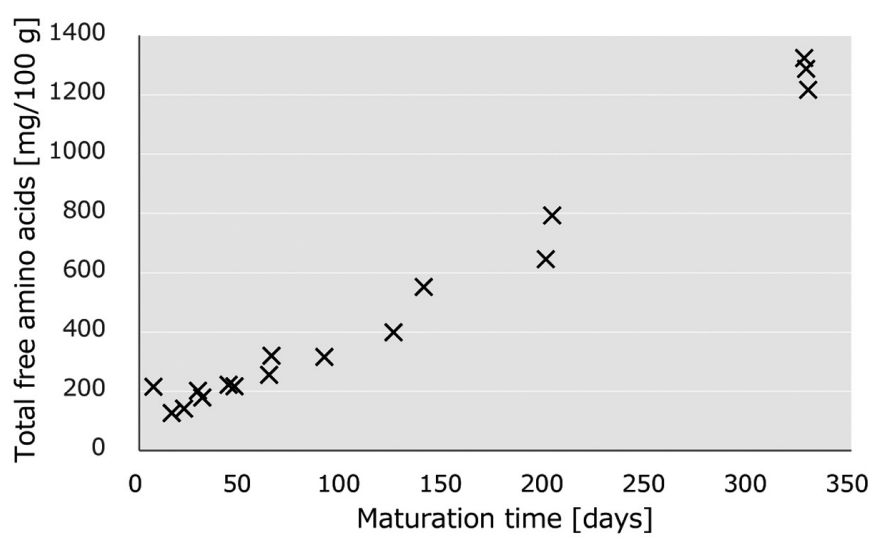

Fig. 1. Scatter plot between maturation time and (A) proteolysis index, and (B) total free amino acids.

(A)

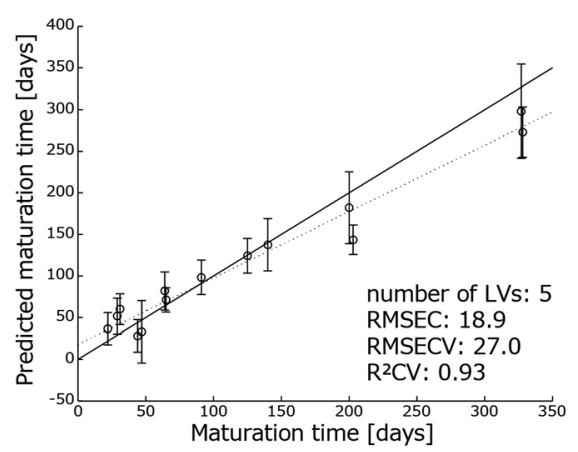

(B)

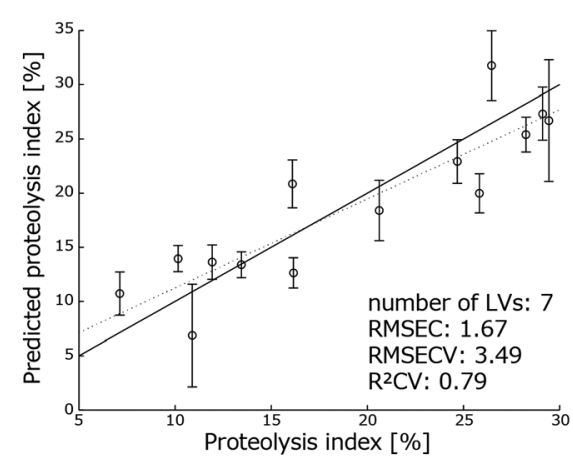

(C)

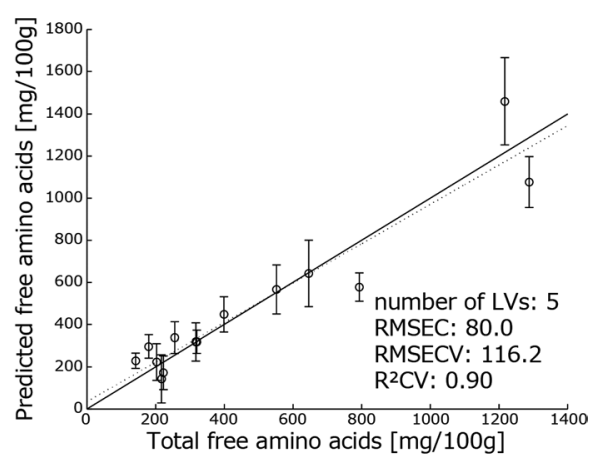

Fig. 2. Scatter plot of predicted value against measured value for (A) maturation time, (B) proteolysis index and (C) total free amino acids. The circles and the error-bars show the average predicted value and standard deviation for the 8 replicate measurements, respectively. Bold lines show y $=\mathrm{x}$ and dotted lines show the linear fit of predicted values. 
Table 1. Cross-validation results to predict maturation time [days], proteolysis index (percentage of soluble nitrogen) and total free amino acids $[\mathrm{mg} / 100 \mathrm{~g}]$ with different preprocessing methods.

Maturation time

\begin{tabular}{lcccc}
\hline & LVs & RMSEC & RMSECV & R $^{2} \mathrm{CV}$ \\
\hline mean-center & 5 & 22.8 & 33.5 & 0.89 \\
\hline normalization + mean-center & 5 & 18.9 & 27.0 & 0.93 \\
\hline autoscale & 4 & 24.8 & 36.0 & 0.87 \\
\hline
\end{tabular}

Proteolysis index

\begin{tabular}{lcccc}
\hline & LVs & RMSEC & RMSECV & R $^{2} \mathrm{CV}$ \\
\hline mean-center & 5 & 3.03 & 4.25 & 0.69 \\
\hline normalization + mean-center & 7 & 1.67 & 3.58 & 0.78 \\
\hline autoscale & 7 & 1.67 & 3.49 & 0.79 \\
\hline
\end{tabular}

Total free amino-acids

\begin{tabular}{lcccc}
\hline & LVs & RMSEC & RMSECV & R $^{2} \mathrm{CV}$ \\
\hline mean-center & 6 & 85.8 & 124.7 & 0.88 \\
\hline normalization + mean-center & 5 & 80.0 & 116.2 & 0.90 \\
\hline autoscale & 3 & 129.9 & 162.6 & 0.80 \\
\hline
\end{tabular}

(A)

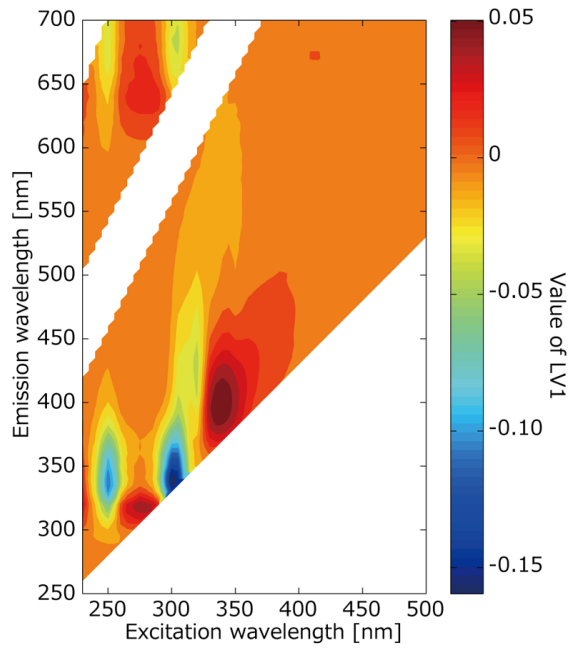

(B)

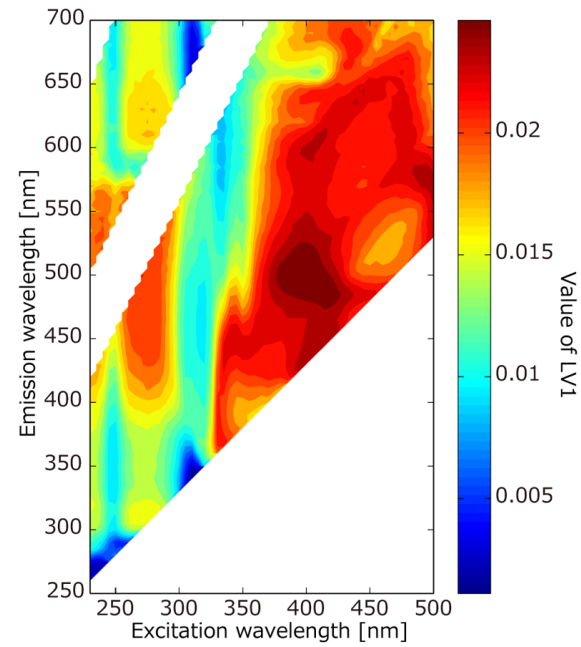

(C)

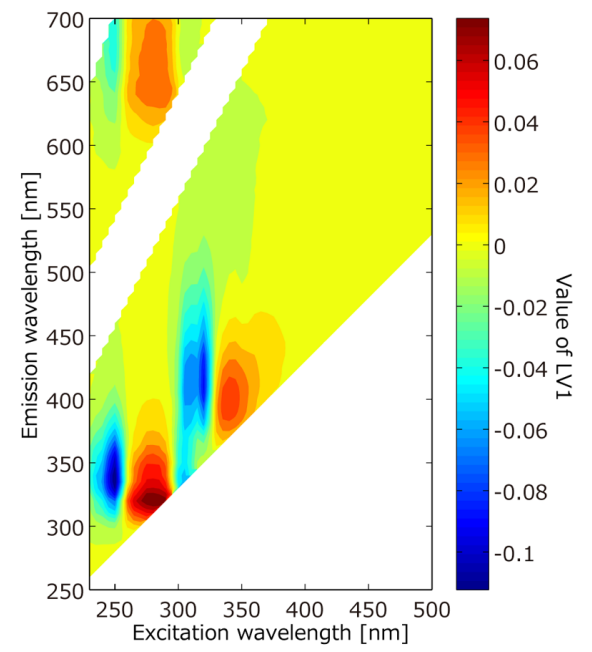

Fig. 3. Coefficients of the 1 st latent variable for the PLS model predicting (A) maturation time, (B) proteolysis index and (C) total amount of free amino acids. A positive peak can be seen at approximately excitation $345 \mathrm{~nm}$ and emission $400 \mathrm{~nm}$ for the coefficient plot of maturation time and free amino acids.

Figure 2 shows the scatter plot of the measured value to the predicted value for maturation time (days), proteolysis index (percentage of soluble nitrogen), and total free amino acids $(\mathrm{mg} / 100 \mathrm{~g})$. The average predicted values and standard deviations for 8 replicate measurements are shown with circles and error-bars, respectively. Normalization followed by mean-center was used for pre-processing the FF data for prediction of maturation time and total free amino acids, and auto-scale was used for prediction of proteolysis index. Maturation time, proteolysis index and total free amino acids were predicted with $\mathrm{R}^{2} \mathrm{CV}$ of $0.93,0.79$ and 0.90 , respectively, and RMSECV of 27.0 days, $3.49 \%$ and $116.2 \mathrm{mg} / 100 \mathrm{~g}$, respectively. Note that the RMSECV is shown in its original units, and the average error of maturation time, proteolysis index and total free amino acids is $8.2 \%, 11.8 \%$ and 
(A)

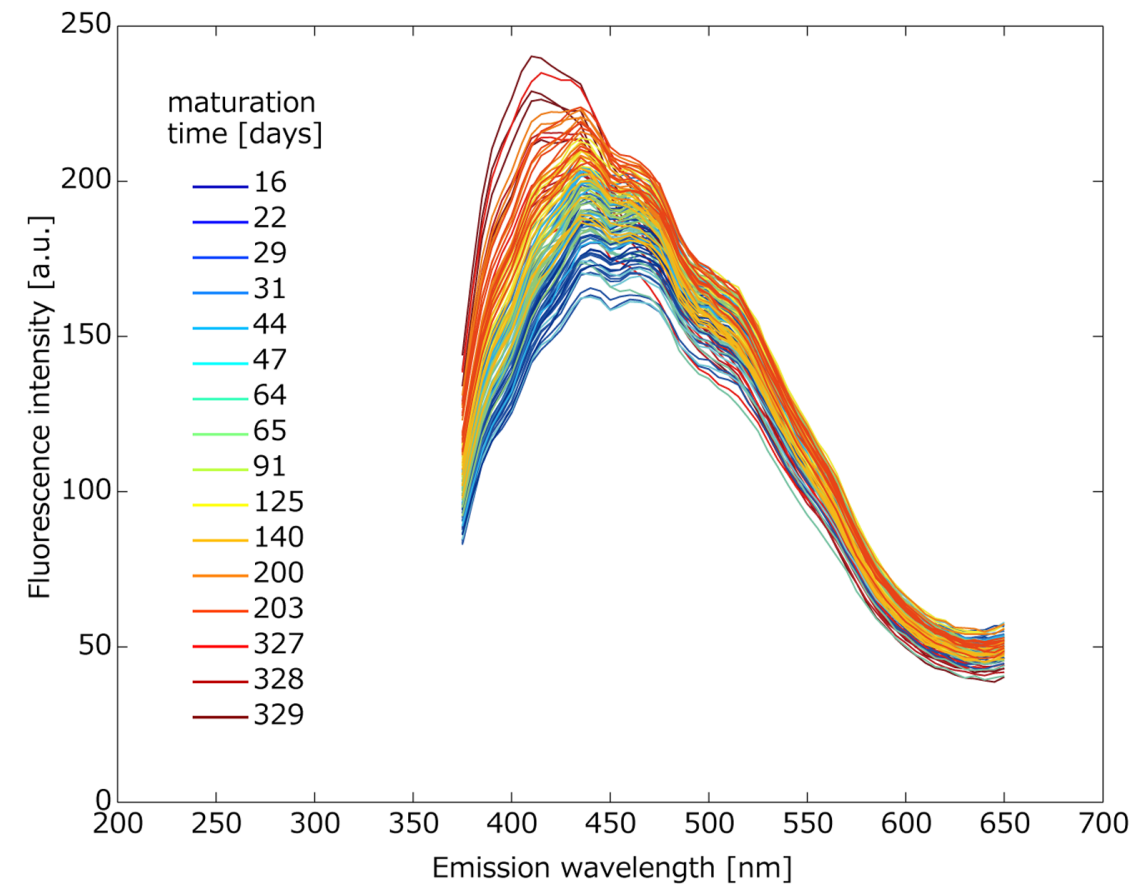

(B)

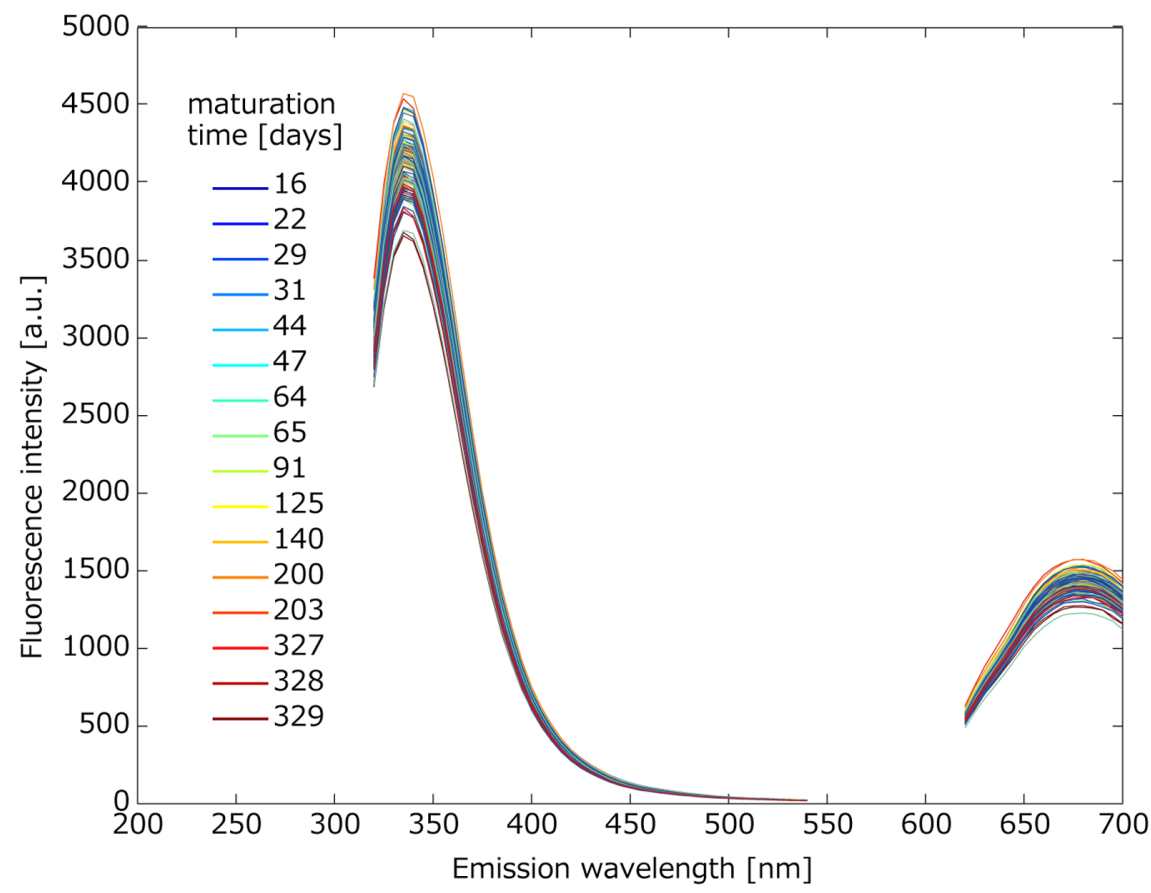

Fig. 4. (A) Fluorescence emission spectra at excitation $345 \mathrm{~nm}$. The fluorescence peak increases with maturation time. (B) Fluorescence emission spectra at excitation $290 \mathrm{~nm}$ is related to aromatic amino acids. The peak intensity is not correlated with maturation time.

$8.8 \%$ of the maximum value, respectively.

The linear fitted line for prediction of maturation time has a gradient that is less than one, and the predicted values for samples with a long maturation time are underestimated. This may be due to the unequal distribution of samples, with many samples with short maturation times, causing the fitted line to be more dependent on the samples with short maturation times. On the other hand, the inability to predict long maturation times could be because chemical change does not progress constantly with long maturation times. Incorporating more samples in this model would help to clarify this point.

Figure 3 shows the coefficients of the first latent variable in the PLS model for predicting maturation time, proteolysis index and total amount of free amino acids. The coefficient plots for prediction of maturation time and free amino acids are similar, with an area of highly positive coefficients around excitation and 
emission wavelengths of $345 \mathrm{~nm}$ and $420 \mathrm{~nm}$, respectively. On the other hand, the wavelength region corresponding to fluorescence of aromatic amino acids, known to have a fluorescence peak at approximately excitation $290 \mathrm{~nm}$ and emission $350 \mathrm{~nm}$ (Andersen and Mortensen, 2008), shows coefficients near zero.

Figure 4A shows the emission spectra of all samples at an excitation wavelength of $345 \mathrm{~nm}$. The fluorescence intensity of this peak increased with prolonged maturation time, and the linear correlation coefficient between maturation time and fluorescence intensity at excitation $345 \mathrm{~nm}$ and emission $420 \mathrm{~nm}$ was 0.81 . Multiple chemical components are known to fluoresce in this wavelength range such as oxidized lipids (Dillard and Tappel, 1971), Maillard reaction products (Birlouez-Aragon et al., 1998; Birlouez-Aragon et al., 2002; Morales et al., 1996; Morales and van Boekel, 1998; Schamberger and Labuza, 2006) and Schiff bases formed between oxidation products and amino groups (Kikugawa and Beppu, 1987). Maillard products are most often linked to the heating process of dairy products, but are known to increase with maturation time (Corzo et al., 2000) and have also been used as an indicator of cheese ripening (Pellegrino et al., 1997). The fluorescence shown in Figure 4A shows multiple overlapping peaks, suggesting that the fluorescence of multiple constituents are increasing in this wavelength range.

Figure 4B shows the fluorescence spectra excited at $290 \mathrm{~nm}$. The fluorescence peak around $345 \mathrm{~nm}$ corresponds to aromatic amino acids. Although there is large variance in fluorescence intensity between samples, there is little correlation between fluorescence intensity and maturation time, and the correlation coefficient between maturation time and fluorescence at excitation $290 \mathrm{~nm}$ and emission $345 \mathrm{~nm}$ is -0.22 . Mazerolles et al. (2001) have used this wavelength range in relation to cheese maturation and indicated that the peak position shifts with prolonged maturing; however, this was not observed in this study. This may be because a wide range of cheese samples produced and matured in different seasons were measured in this study, leading to large inter-sample variance that affected the fluorescence intensity at this wavelength.

\section{Conclusions}

Three indices of cheese maturation were predicted from the FF data: maturation time, proteolysis index and total free amino acids. A PLS regression model was calculated, showing $\mathrm{R}^{2} \mathrm{CV}$ values of $0.93,0.79$ and 0.90 for prediction of maturation time, proteolysis index and total free amino acids, respectively. The wavelength range corresponding to oxidized lipids and Maillard products showed increasing fluorescence with maturation time; however, fluorescence corresponding to aromatic amino acids showed low correlation. Since the intensity of fluorescence corresponding to oxidized lipids and Maillard products is only about $5 \%$ of the intensities of the aromatic amino acids, the dominant fluorophors in cheese, it has a low possibility of being targeted with conventional fluorescence. In this respect, FF has an advantage over conventional fluorescence in that it is a non-targeted approach.

FF measurement is much quicker and easier than chemical analysis, and the measurement can be performed non-destructively with the optical fibers of hand-held devices. Although more data needs to be incorporated into the PLS model for increased robustness and reliability, the FF has shown to be a promising method to measure the maturation of cheese.

\section{References}

Addeo, F., Garro, G., Intorcia, N., Pellegrino, L., Resmini, P., and Chianese, L. (1995). Gel electrophoresis and immunoblotting for the detection of casein proteolysis in cheese. J. Dairy Res., 62, 297-309.

Andersen, C. M. and Mortensen, G. (2008). Fluorescence spectroscopy: A rapid tool for analyzing dairy products. J. Agric. Food Chem., 56, 720729.

Andersen, C. M., Vishart, M., and Holm, V. K. (2005). Application of fluorescence spectroscopy in the evaluation of light-induced oxidation in cheese. J. Agric. Food Chem., 53, 9985-9992.

Birlouez-Aragon, I., Nicolas, M., Metais, A., Marchond, N., Grenier, J., and Calvo, D. (1998). A rapid fluorimetric method to estimate the heat treatment of liquid milk. Int. Dairy J., 8, 771-777.

Birlouez-Aragon, I., Sabat, P., and Gouti, N. (2002). A new method for discriminating milk heat treatment. Int. Dairy J., 12, 59-67.

Christensen, J., Povlsen, V. T., and Sorensen, J. (2003). Application of fluorescence spectroscopy and chemometrics in the evaluation of processed cheese during storage. J. Dairy Sci., 86, 1101-1107.

Corzo, N., Villamiel, M., Arias, M. a., Jiménez-Pérez, S., and Morales, F. J. (2000). The maillard reaction during the ripening of manchego cheese. Food Chem., 71, 255-258.

Dillard, C. J. and Tappel, A. L. (1971). Fluorescent products of lipid peroxidation of mitochondria and microsomes. Lipids, 6, 715-721.

Dufour, E., Devaux, M. F., Fortier, P., and Herbert, S. (2001). Delineation of the structure of soft cheeses at the molecular level by fluorescence spectroscopy - relationship with texture. Int. Dairy J., 11, 465-473.

Dufour, E., Mazerolles, G., Devaux, M. F., Duboz, G., Duployer, M. H., and Riou, M. N. (2000). Phase transition of triglycerides during semihard cheese ripening. Int. Dairy J., 10, 81-93.

Dufour, E. and Riaublanc, A. (1997). Potentiality of spectroscopic methods for the characterisation of dairy products. I. Front-face fluorescence study of raw, heated and homogenised milks. Lait, 77, 657-670.

Fenelon, M. A., O'Connor, P., and Guinee, T. P. (2000). The effect of fat content on the microbiology and proteolysis in cheddar cheese during ripening. J. Dairy Sci., 83, 2173-2183.

Fujita, K., Tsuta, M., Kokawa, M., and Sugiyama, J. (2010). Detection of deoxynivalenol using fluorescence excitation-emission matrix Food Bioprocess Technol., 3, 922-927.

Guimet, F., Ferre, J., Boque, R., and Rius, F. X. (2004). Application of unfold principal component analysis and parallel factor analysis to the exploratory analysis of olive oils by means of excitation-emission matrix fluorescence spectroscopy. Anal. Chim. Acta, 515, 75-85.

Henry, R. C., Park, E. S., and Spiegelman, C. H. (1999). Comparing a new 
algorithm with the classic methods for estimating the number of factors. Chemometrics Intellig. Lab. Syst., 48, 91-97.

Herbert, S., Riou, N. M., Devaux, M. F., Riaublanc, A., Bouchet, B., Gallant, D. J., and Dufour, E. (2000). Monitoring the identity and the structure of soft cheeses by fluorescence spectroscopy. Lait, 80, 621-634.

Jiang, J. K., Wu, J., and Liu, X. H. (2010). Fluorescence properties of lake water. Spectrosc. Spect. Anal., 30, 1525-1529.

Jin, Y. K. and Park, Y. W. (1995). Effects of aging time and temperature on proteolysis of commercial goat milk cheeses produced in the united states. J. Dairy Sci., 78, 2598-2608.

Kapoor, R., Lehtola, P., and Metzger, L. E. (2004). Comparison of pilotscale and rapid visco analyzer process cheese manufacture. J. Dairy Sci., 87, 2813-2821.

Kapoor, R. and Metzger, L. E. (2008). Process cheese: Scientific and technological aspects-a review. Comprehensive Reviews in Food Science and Food Safety, 7, 194-214.

Karoui, R., Dufour, É., and De Baerdemaeker, J. (2007). Front face fluorescence spectroscopy coupled with chemometric tools for monitoring the oxidation of semi-hard cheeses throughout ripening. Food Chem., 101, 1305-1314.

Karoui, R., Dufour, E., Pillonel, L., Picque, D., Cattenoz, T., and Bosset, J. O. (2004). Determining the geographic origin of emmental cheeses produced during winter and summer using a technique based on the concatenation of mir and fluorescence spectroscopic data. Eur. Food Res. Technol., 219, 184-189.

Kikugawa, K. and Beppu, M. (1987). Involvement of lipid oxidation products in the formation of fluorescent and cross-linked proteins. Chem. Phys. Lipids, 44, 277-296.

Kuchroo, C. N. and Fox, P. F. (1982). Soluble nitrogen in cheddar cheese: Comparison of extraction procedures. Milchwissenschaft, 37, 331-335.

Lee, B. H., Laleye, L. C., Simard, R. E., Munsch, M. H., and Holley, R. A. (1990). Influence of homofermentative lactobacilli on the microflora and soluble nitrogen components in cheddar cheese. J. Food Sci., 55, 391397.

Mazerolles, G., Devaux, M. F., Duboz, G., Duployer, M. H., Riou, N. M., and Dufour, E. (2001). Infrared and fluorescence spectroscopy for monitoring protein structure and interaction changes during cheese ripening. Lait, 81, 509-527.

Ministry of Agriculture, Forestry and Fisheries of Japan (2013). Demand and supply of cheese in year 2012. Tokyo, Japan

Moller, J. K. S., Parolari, G., Gabba, L., Christensen, J., and Skibsted, L. H. (2003). Monitoring chemical changes of dry-cured parma ham during processing by surface autofluorescence spectroscopy. J. Agric. Food Chem., 51, 1224-1230.

Morales, F. J., Romero, C., and Jiménez-Pérez, S. (1996). Fluorescence associated with maillard reaction in milk and milk-resembling systems. Food Chem., 57, 423-428.

Morales, F. J. and van Boekel, M. A. J. S. (1998). A study on advanced maillard reaction in heated casein/sugar solutions: Colour formation. Int. Dairy J., 8, 907-915.

Pellegrino, L., Battelli, G., Resmini, P., Ferranti, P., Barone, F., and Addeo, F. (1997). Effects of heat load gradient occurring in moulding on characterization and ripening of grana padano. Lait, 77, 217-228.

Piska, I. and Štětina, J. (2004). Influence of cheese ripening and rate of cooling of the processed cheese mixture on rheological properties of processed cheese. J. Food Eng., 61, 551-555.

Sadecka, J. and Tothova, J. (2007). Fluorescence spectroscopy and chemometrics in the food classification - a review. Czech. J. Food Sci., 25, 159-173.

Schamberger, G. P. and Labuza, T. P. (2006). Evaluation of front-face fluorescence for assessing thermal processing of milk. J. Food Sci., 71, C69-C74.

Sikorska, E., Glisuzynska-Swiglo, A., Insinska-Rak, M., Khmelinskii, I., De Keukeleire, D., and Sikorski, M. (2008). Simultaneous analysis of riboflavin and aromatic amino acids in beer using fluorescence and multivariate calibration methods. Anal. Chim. Acta, 613, 207-217.

Trivittayasil, V., Tsuta, M., Imamura, Y., Sato, T., Obata, A., Otomo, H., Kokawa, M., Sugiyama, J., Fujita, K., and Yoshimura, M. (2015). Fluorescence fingerprint as an instrumental assessment of the sensory quality of tomato juices. J. Sci. Food Agric. (in press)

Yin, C. L., Li, H., Ding, C. H., and Wang, H. (2009). Preliminary investigation on variety, brewery and vintage of wines using threedimensional fluorescence spectroscopy. Food Sci. Technol. Res., 15, $27-$ 38.

Yoshimura, M., Sugiyama, J., Tsuta, M., Fujita, K., Shibata, M., Kokawa, M., Oshita, S., and Oto, N. (2014). Prediction of aerobic plate count on beef surface using fluorescence fingerprint. Food Bioprocess Technol., 7, 1496-1504. 